(Aus dem Petrograder Staatsinstitut für Gehirnforschung [Direktor:

Akademiker W. M. Bechterew].)

\title{
Pathologisch-anatomische Veränderungen der Gehirnrinde bei der Cholera asiatica.
}

(Vorläufige Mitteilung.)

\author{
Von \\ Dr. I. L. Pines, \\ Assistent am Institut. \\ Mit 4 Textabbildungen. \\ (Eingegangen am 1\%. Juni 1922.)
}

Während die pathologisch-anatomischen Veränderungen verschiedener Organe bei der Cholera vielfach studiert und beschrieben worden sind, ist eine nur ganz winzige Zahl von Publikationen über die gleichen Veränderungen des Zentralnervensystems und speziell der Gehirnrinde erschienen und auch stehen diese Arbeiten in manchen Punkten im Widerspruch zueinander. Um den oft sehr markanten Erscheinungen, die auf Reizung und Lähmung des Zentralnervensystems hinweisen, eine genügend erforschte pathologisch-anatomische Grundlage zu schaffen, sind weitere Arbeiten auf diesem Gebiete notwendig. Ohne hier auf die Literatur einzugehen, möchte ich die eigenen Beobachtungen während der letzten Choleraepidemie in Rußland, Herbst 1921, die ich an der pathologisch-anatomischen Abteilung (Chef: Prof. W. Afanasieff) des Bakteriologischen Institutes (Direktor: Prof. $M$. Stutzer) in Woronesch zu machen Gelegenheit hatte, kurz resumieren.

Im ganzen wurden 5 Gehirne darauf untersucht. Sie stammten von Patienten im 30. - 40. Jahresalter. Alle starben im Stadium des Choleratyphoids. (Man kann vermuten, daß in diesem Stadium die größten, die am meisten konstanten und für die Cholera am meisten charakteristischen, wenn es solche überhaupt gäbe, Zerstörungen des Zentralnervensystems zum Ausdruck kommen müssen).

Die anamnestischen und klinischen Angaben waren infolge der Epidemieverhältnisse mangelnde.

Es wurde die einfachste Technik gewählt:

Stückchen zum Studium wurden von den Lobi frontal., zentral., temporal., parietal., occipitales des Großhirns, sowie vom Kleinhirn entnommen (Rinde und Mark);

Härtung in $4 \%$ Formalinlösung; 
Einbettung in Paraffin;

Anfertigung der Schnitte am Schlittenmikrotom;

Färben mit Karmin, Hämatoxylin-Eosin, Methylenblau und nach Van-Gieson.

Die Veränderungen sind, im Grunde genommen, in allen Gehirnen die gleichen; sie variieren nur nach Intensität. Ich werde darum hier nicht jedes Gehirn einzeln beschreiben, sondern mich auf die allgémeine Beschreibung der Veränderungen beschränken. Dort, wo die Ver-

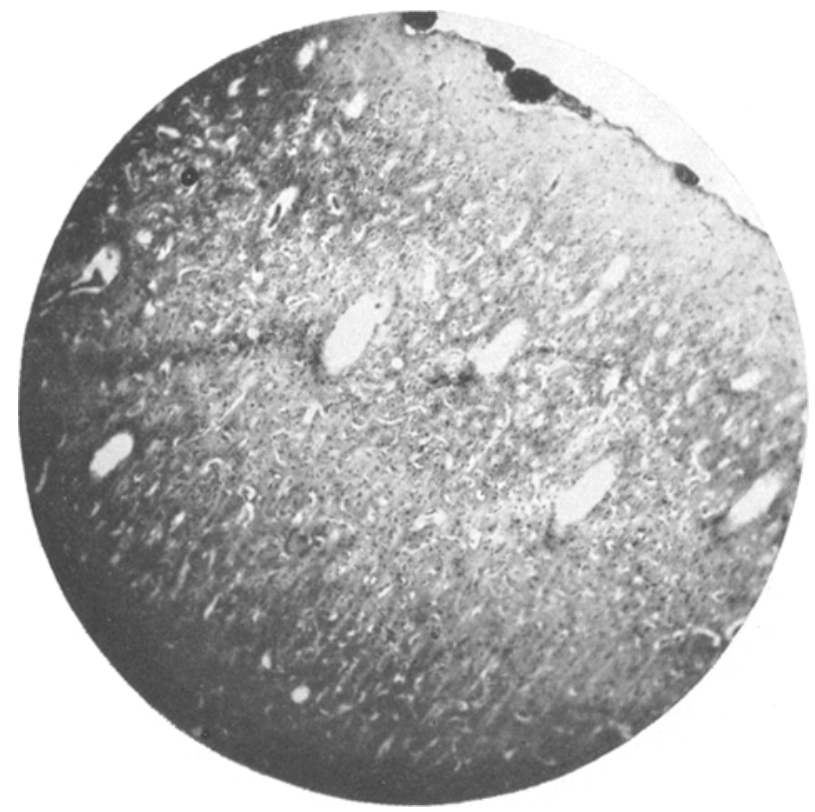

Abb. 1. Mikroskop Reichert. Obj. 3. Ocul. 1. Hyperämie der Pia mater. Sièbartiges Aussehen der Zellschichten (état criblé). Intaktbleiben der Molekularschicht und der weiben Substanz.

änderungen am meisten ausgesprochen sind, machen sie sich schon bei kleiner Vergrößerung bemerkbar: die Rinde hat hier ein siebartiges Aussehen, sie besteht vorzugsweise aus kleinen leeren Räumchen (état criblé der Franzosen). (Abb. 1.) In diesen Räumchen, die als Folge des Gehirnödems betrachtet werden dürfen, sind hie und da amorphe Massen, die Nervenzellendetritus oder stark entartete und schlecht färbbare Nervenzellen darstellen, anzutreffen. Zwischen den Räumchen sind neben degenerierten Zellen noch verhältnismäßig gut erhaltene zu finden. Wo die Veränderungen weniger ausgesprochen sind, merkt man Schwellung und Trübung der Nervenzellen, Wanderung des Kernes an die Peripherie, schlechte Färbbarkeit der Zellen und Zellenfortsätze. Es macht sich eine Tendenz zur Vergrößerung der Pericellularräume 
geltend: um die Zellen herum erscheinen leere Räume, die Zellenkapseln sind wie ausgezogen und die Zellen füllen nicht den ganzen Zellenraum aus. (Abb. 2.) Parallel mit dem Fortschreiten der Zellentartung vergrößern sich die sie umgebenden leeren Räume bis zur Bildung des oben erwähnten état criblé dort, wo die Degeneration am meisten fortgeschritten ist. Diese kleinen leeren Räume stellen ein begleitendes charakteristisches Merkmal der Nervenzellendegeneration dar; in der weißen Substanz sind sie nicht anzutreffen, und in den äußeren Rindenpartien, wo es

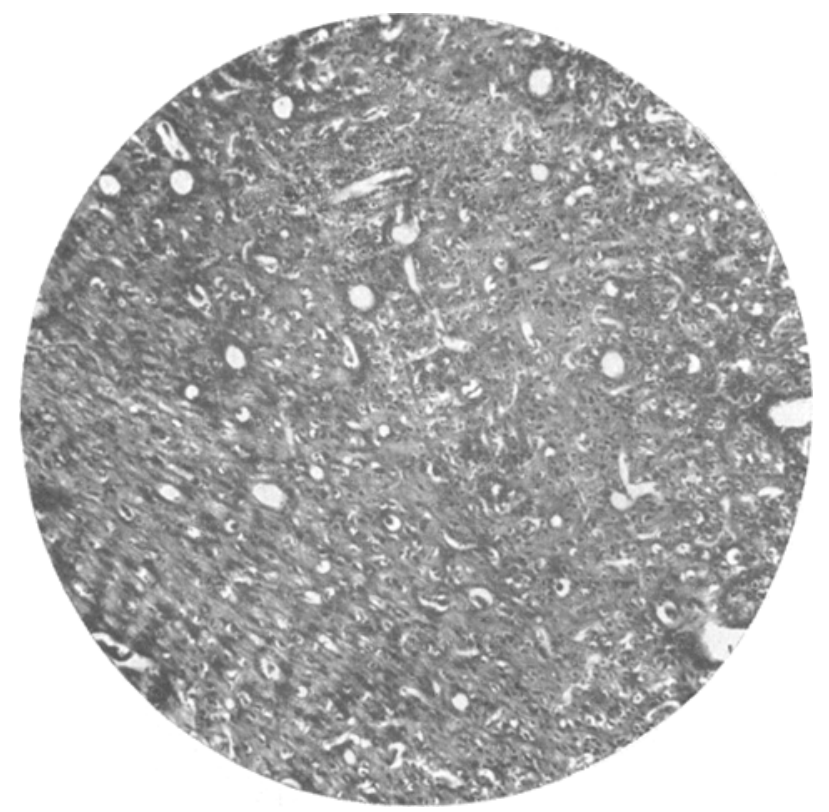

Abb. 2. Mikroskop Reichert. Obj. 3. Ocul. 2. Erweiterung der Pericellularräume. Hämatoxylinpräparat.

weniger Zellen gibt, sind sie auch weniger zahlreich. Auf Grund der von. mir angefertigten Präparate ist es unmöglich zu behaupten, da $\beta$ die Veränderungen bestimmte Rindengebiete bevorzugen: es scheint, daß es sich um einen diffusen Prozeß handelt, um Veränderungen, die die gesamte Rinde als solche ergreifen. Was die Intensität der Veränderungen anbelangt, so variiert sie stark: in einigen Gehirnen sind die Veränderungen unbedeutend, in den anderen nimmt die Zerstörung der Nervensubstanz große Dimensionen an. Es ist eine individuelle Disposition, eine individuell verschiedene Reaktionsfähigkeit des Gehirnes anzunehmen, eventuell auch eine verschiedene Intensität der Infektion. Worin eine solche Disposition besteht, ist beim heutigen Stande unseres Wissens schwer bestimmt zu beantworten. 
In den Nervenzellen des Kleinhirnes ist es mir nicht gelungen, irgendwelche nennenswerte Veränderungen festzustellen; auch die Purkinjesche Zellen bleiben normal, nur hie und da zeigen sie eine leichte Trübung.

Also, die am meisten für das Choleratyphoid typische Veränderung ist die Degeneration der Nervenzellen der Großhirnrinde und das sie begleitende Ödem. (Abb. 3 u. 4.)

Was die Nervenfasern anbelangt, so ist es mir hier nicht gelungen, irgendwelche Veränderungen festzustellen.

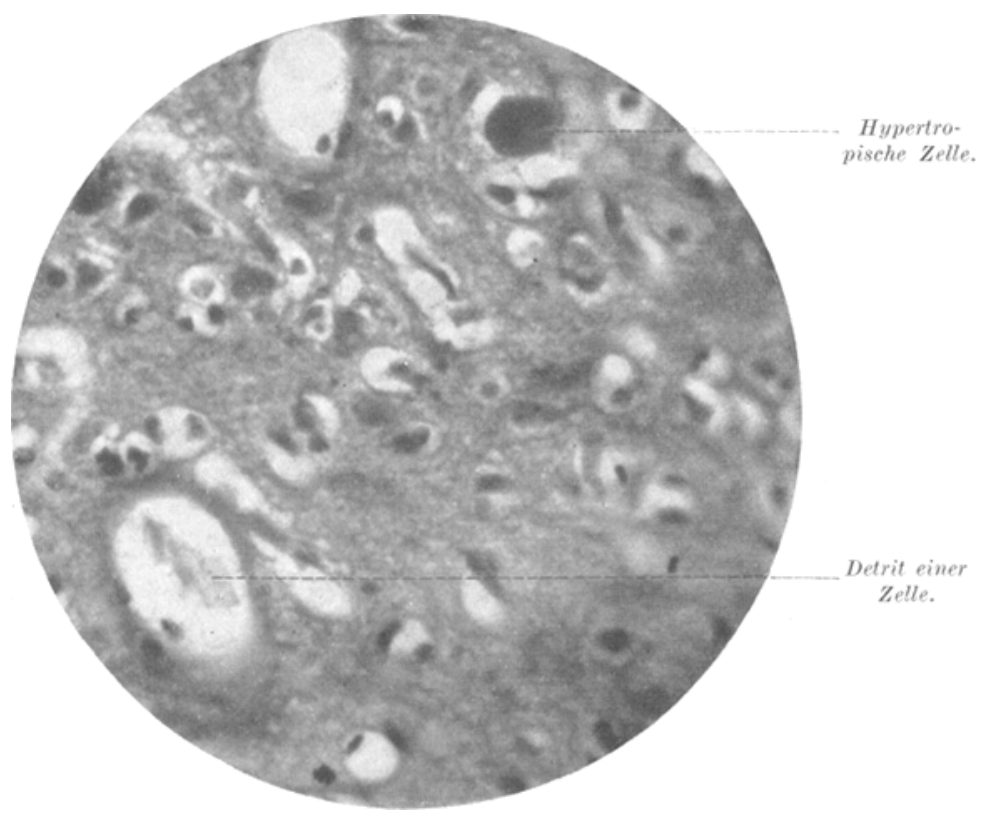

Abb. 3. Mikroskop Reichert. Obj. 7. Ocul. 1. Hämatoxylinpräparat.

Auch das Gliagewebe bleibt intakt. Man kann hier nicht von einer Vergrößerung der einzelnen Elemente, nicht von einer Zahlvermehrung derselben sprechen. Nirgends ist eine Ansammlung der Neurogliakerne zu finden; hie und da sind ein, zwei bis drei mit Hämatoxylin stark färbbare Kerne in den Zellkörpern, sowie auch in den Pericellularräumen anzutreffen.

Was die Gefäße anbelangt, so sind speziell die Gefäße der Pia mater teilweise mit Blut überfüllt. Das Gehirn selbst zeigt keine deutliche Hyperämie, keine Gefäßwandveränderungen, nur hie und da eine Verbreiterung der Perivasculärräume, aber keine Emigration lymphoider Elemente, keine entzündliche Exsudation und Ansammlung von Blutelementen.

Resumierend: die anatomischen Veränderungen des Gehirnes, die man beim Choleratyphoid zu beobachten bekommt, stellen einen 
diffusen Prozeß primärer Degeneration und Nekrose der Nervenzellen der Großhirnrinde dar. Vielleicht zeigt sich eine gewisse Spezifizität der Cholerawirkung darin, daß in erster Reihe und hauptsächlich die physikalisch-chemischen, osmotischen Verhältnisse der Zellen leiden, was zur Ödembildung um sie herum führt; letztere kann nicht durch Zirkulationsveränderungen erklärt werden, da die mikroskopischen Bilder keine genügenden Anhaltspunkte dafür bieten. Es sind keine gefäßentzündliche, keine reaktiv-hyperplastische Prozesse im Gehirn beim

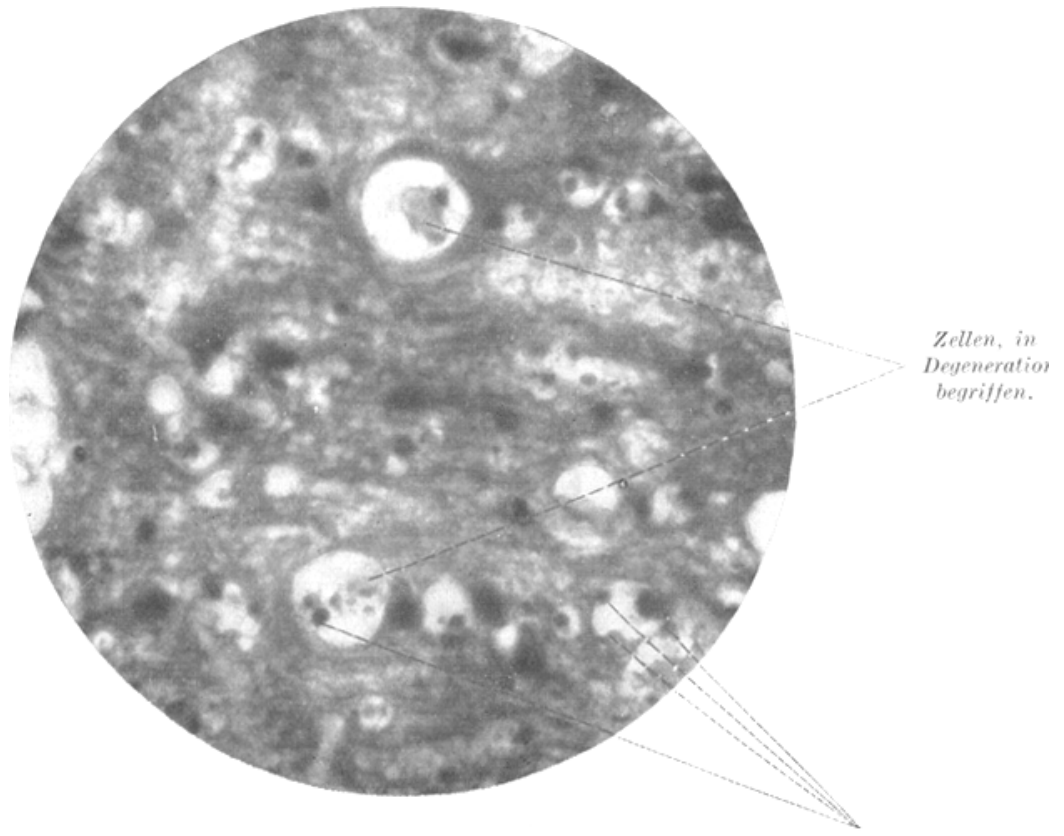

Abb. 4. Mikroskop Reichert. Obj. 7. Ocul. 1. Zellendegeneration. Neuronophagen Neuronophagie. Karmiupräparat.

Choleratyphoid vorhanden. Die rein degenerativen Prozesse sind durch eine Intoxikation mit Choleragift (Toxine) erklärbar. Diese Anschauung findet sich in vollem Einklange mit dem heutigen Stande unseres Wissens über die Cholera.

Herm W. Afanasieff, Professor der pathologischen Anatomie an der gewes. Universität von Dorpat, der die Liebenswürdigkeit hatte, alle von mir angefertigten Präparate zu durchmustern, danke ich verbindlichst. Auch wurden die Präparate am 28. XII. 1921 an der Sitzung der medizinischen Pirogowgesellschaft der Universität von Woronesch und am 29. V. 1922 an der wissenschaftlichen Konferenz des Petrograder Staatsinstitutes für Gehirnforschung von mir demonstriert. 